Technical Note

\title{
Stratospheric Aerosol and Gas Experiment (SAGE) from SAGE III on the ISS to a Free Flying SAGE IV Cubesat
}

\author{
John P. Leckey ${ }^{*+}+\mathbb{D}$, Robert Damadeo ${ }^{+}(\mathbb{D})$ and Charles A. Hill ${ }^{+(\mathbb{C})}$ \\ NASA Langley Research Center, Hampton, VA 23681, USA; robert.damadeo@nasa.gov (R.D.); \\ charles.a.hill@nasa.gov (C.A.H.) \\ * Correspondence: john.p.leckey@nasa.gov \\ † These authors contributed equally to this work.
}

check for updates

Citation: Leckey, J.P.; Damadeo, R.; Hill, C.A. Stratospheric Aerosol and Gas Experiment (SAGE) from SAGE III on the ISS to a Free Flying SAGE IV Cubesat. Remote Sens. 2021, 13, 4664. https://doi.org/ $10.3390 /$ rs13224664

Academic Editor: Jose Moreno

Received: 27 September 2021 Accepted: 15 November 2021 Published: 19 November 2021

Publisher's Note: MDPI stays neutral with regard to jurisdictional claims in published maps and institutional affiliations.

Copyright: (C) 2021 by the authors. Licensee MDPI, Basel, Switzerland. This article is an open access article distributed under the terms and conditions of the Creative Commons Attribution (CC BY) license (https:/ / creativecommons.org/licenses/by/ $4.0 /)$.

\begin{abstract}
The Stratospheric Aerosol and Gas Experiment III (SAGE III) on the International Space Station (ISS) is widely accepted as a stable source for high-quality stratospheric ozone, aerosol, and water vapor measurements since it was installed on the ISS in 2017. The ISS is a unique platform that provides access for hosted payloads while furnishing infrastructure for power, uplink, downlink, etc. for instrument operations. The opportunities, risks, and challenges from operating on the ISS are described in addition to comprehensive lessons learned. In addition, SAGE IV is presented as an option for the future of the SAGE lineage where the lessons learned from SAGE III and technological advances have enabled the instrument to fit into a $6 \mathrm{U}$ CubeSat yielding a significantly smaller and cheaper form-factor to preserve the continuity of critical atmospheric measurements.
\end{abstract}

Keywords: stratosphere; ozone; aerosol; ISS; SAGE III; SAGE IV

\section{Introduction to SAGE Instruments}

The SAGE missions are a series of remote sensing instruments deployed in Low-Earth Orbit (LEO) with the intent of observing key atmospheric constituents, in particular aerosol, ozone, and water vapor. SAGE I [1,2], operational between 1979 and 1981, successfully used the technique of solar occultation (i.e., using the Sun as a light source through long atmospheric path lengths to measure weakly absorbing/scattering species) to make some of the first vertically resolved measurements of stratospheric aerosol and ozone. These measurements contributed to the earliest assessments of the impact of chlorofluorocarbons on the ozone layer [3] and the passing of the Montreal Protocol to phase out, and eventually ban, the use of these substances. SAGE II [4,5], active from 1984 until its decommissioning in 2005, provided the longest single-instrument data records of stratospheric ozone, aerosol, and water vapor. These data have been crucial to assessing the efficacy of the Montreal Protocol [6]. SAGE II also observed one of the largest volcanic eruptions of the 20th century, Mount Pinatubo in 1991, and its impacts on the stratosphere [7]. The SAGE III instruments [8] were built as a trio, with one to fly on an independent spacecraft, one that was meant to be mounted to the International Space Station (ISS), and one to be used as a flight of opportunity. For various reasons, the free-flyer was active from 2002-2005 while the other two were put into storage until SAGE III/ISS was launched and installed in 2017.

Over the past few years, SAGE III/ISS has demonstrated an ability to provide highquality measurements of stratospheric ozone [9], aerosol [10], and water vapor [11,12]. These atmospheric species are of particular importance. Stratospheric ozone, for example, is a targeted observable in the 2017 Decadal Survey [13], and NASA is mandated to monitor the health of the ozone layer under the Clean Air Act as amended [14]. Aerosol is a designated observable under the Decadal Survey because it is a key input to climate models, has an important role in atmospheric chemistry [15], and is a large source of uncertainty to Earth's energy balance [16-18]. Water vapor is another key component of Earth's energy budget [16], a key driver of stratospheric chemistry [19], and a tracer 
for stratospheric circulation and stratosphere-troposphere exchange [20-23]. Despite the importance of these constituents, there is uncertainty about the future of availability of measurements and data continuity. While the SAGE III mission is in extended operation, it is important to think about what instruments can be deployed in the future to ensure proper continuity of these critical data records.

\section{ISS as a Platform for SAGE III}

Unlike previous SAGE instruments, which were mounted on free-flying spacecraft of different sizes, SAGE III on the ISS operates from a unique platform that furnishes both benefits and challenges. The ISS sits in a mid-inclination $\left(51.6^{\circ}\right)$ low-altitude orbit $(\approx 400 \mathrm{~km})$ similar to SAGE II ( $57^{\circ}$ inclination at $\left.650 \mathrm{~km}\right)$, which is ideal for solar occultation observations. While SAGE instruments do not create excessive amounts of data, the ISS provides communications through the Tracking and Data Relay Satellites (TDRS) and the Space Network not only for large volumes of data to downlink for archiving and processing, but also often real-time streaming of telemetry with minimal latency. Additionally, as an inhabited space station there is the possibility of servicing the aging auxiliary hardware, such as when a Battery Charge/Discharge Unit (BCDU) failure in the ExPRESS Logistics Carrier-4 (ELC-4) power system interrupted operational power to the SAGE III instrument payload for one month in September-October 2019. A spacewalk to replace the BCDU was completed on 18 October 2019 by astronauts Jessica Meir and Christina Koch. However, as a very large, multi-module habitat with regularly visiting vehicles, the ISS provides unique challenges to a remote sensing instrument. These challenges generally fall into one of two categories: interruptions to operations and pointing anomalies.

\subsection{Operational Interruptions}

By its nature as a manned space station, there is a routine schedule of visiting spacecraft to the ISS. Like most instruments, SAGE is sensitive to its environment, and visiting vehicles have a tendency to temporarily contaminate that environment through the release of propellants and molecular outgassing and desorption from spacecraft exteriors. Because of the unique platform, SAGE III/ISS has two Contamination Monitoring Packages (CMPs) that are designed to measure molecular contaminant flux from eight directions covering the $2 \pi$ steradians on the payload side of the Expedite the Processing of Experiments to the Space Station (ExPRESS) payload adapter [24]. Additionally, all SAGE instruments have a contamination door containing an optically transparent window meant to cover the optics prior to and during launch. Previous SAGE instruments opened this door after launch and left it in that position, but SAGE III/ISS routinely opens and closes this door as vehicles come and go and contaminant fluxes wax and wane. The contamination door is meant to prevent contaminants from entering the scan head and optical train while allowing science measurements to continue through the optical window with a reduction in measurement signal-to-noise ratio as contamination adsorbs on the window. The relative nature of occultation atmospheric transmission measurements permits this functionality, and window noise is reflected in the species product error bars. Through 5 November 2021, SAGE III/ISS executed 42,112 solar occultation events and 5518 lunar occultation events, with more science events being collected every orbit. The contamination door has been open for only $58.1 \%$ of solar occultation events and $55.0 \%$ of lunar occultation events. While science operations with a somewhat diminished signal-to-noise ratio have taken place with the contamination door closed, operations are halted during active docking/undocking maneuvers and nearby space walks, or during periods of unusually high contamination as detected by the CMPs. Through 5 November 2021, 736 solar occultation opportunities and 269 lunar occultation opportunities have been lost to SAGE operational pauses from these ISS activities. A further 848 solar opportunities and 164 lunar opportunities have been lost to ISS power outages at the SAGE III ELC-4 site. Other sources of operational interruptions are less direct and unintentional. Because of its size and configuration, and because of the changing orbital solar beta angle, there are some modules that routinely obstruct SAGE's 
view of the Sun. Visiting vehicles act similarly. Sometimes these obstructions occur for a short enough period of time that the instrument continues to function normally, while at other times it fails to reacquire the Sun and ceases an active science event. This both reduces the total number of science events collected and presents additional challenges to identify these events in processing, such as modifying the science retrieval algorithm to exclude the use of data obscured by station components to the extent possible [25]. Through 5 November 2021, 2507 solar occultation opportunities and 715 lunar occultation opportunities were lost to ISS line-of-sight obstructions.

\subsection{Pointing Anomalies}

When it comes to solar occultation measurements, pointing knowledge is critical. This is primarily because the Sun is not a uniform light source and, while pointing uncertainty in the atmosphere degrades vertical resolution, pointing uncertainty on the face of the Sun results in radiometric uncertainty. As such, a significant effort is expended to characterize and calibrate the mechanisms that control the rotating scan head or the pivoting scan mirror. Even still, there are certain assumptions that are made during data processing. For example, the science processing algorithm assumes that the spacecraft maintains attitude such that the primary axis of the science instrument is oriented towards nadir. For past SAGE instruments, onboard free-flying spacecraft, this was a reasonable assumption. However, the ISS is, relatively speaking, an enormous spacecraft composed of multiple modules that can bend and flex relative to each other. Additionally, the overall attitude of the ISS changes temporarily as visiting vehicles come and go, modifying the mass distribution and drag profile of the station. The primary SAGE III/ISS science instrument is mounted to a Hexapod assembly that can alter the orientation of the instrument to help compensate for changes in the overall orientation of the ISS. However, this does not account for movements of the SAGE payload from station bending modes. Instead, SAGE III/ISS has a Disturbance Monitoring Package (DMP), which is a three-axis ring laser gyro mounted at the base of the Hexapod, to help measure these rotational changes so that they can be accounted for during the science retrieval algorithm.

\section{ISS Lessons Learned for Future Payloads}

During a project's life cycle, specialized knowledge and experience are ideally documented with review and other process controls ensuring accuracy, clarity, traceability, and accessibility. A typical document product includes engineering drawings, bills of material, schematics, traveler logs, quality assurance certifications, reports and manuals, theoretic basis and control documents, requirements and verification artifacts, schedule and budget records, risk and project management plans, milestone review material, procedures, photographs, waivers, and nonconformance reports, along with electronic source files, software, and firmware contained in version control repositories with commit logs and a ticketing system. To stimulate a culture of learning the SAGE III/ISS project also tracked Lessons Learned in a database. These lessons included cases where the project team executed well and cases where execution could have been improved. A culture of learning gains knowledge from both successful and unsuccessful work and captures that knowledge in a consumable way. Specific lessons are then generalized and ultimately translated into future best practices that can mature the overall project management processes. NASA project management is the dynamism for deriving value from taxpayer money. Tremendous value is lost to NASA when knowledge and experience are neglected or abandoned through attrition. Knowledge captures pertinent to external payloads aboard the ISS generally fell into the categories of Hardware, Software, Documentation, Science, Mission Operations, and Personnel lessons. Selected examples of general interest follow.

\subsection{Hardware: The Anticipated ISS Pitch Bias Was Mutable}

The ISS orbits in a Torque Equilibrium Attitude (TEA) to limit the rate at which the station's control moment gyroscopes accumulate the angular momentum induced by 
external torques such as atmospheric drag and gravity gradient torques. TEA will change with visiting vehicles and with the arrival of new modules. Several external payloads have been designed with fixed instrument attitude offsets narrowly tailored to the latest ISS attitude assumptions. For instance, the SAGE III static Hexapod lower wedge offset was determined during the Assembly, Integration, and Test (AI\&T) Phase D based on the contemporary ISS configuration as well as the imminent arrival of the Nauka module. The static wedge pitches the Hexapod at its base by $7^{\circ}$ in anticipation of the TEA needed to support Nauka. Because the launch of this module was delayed several years past the February 2017 arrival of SAGE III/ISS, the Hexapod typically operated closer to its outer range of motion envelope during the Prime Mission. The SAGE III Hexapod has a $\pm 8^{\circ}$ range of motion in the pitch and roll directions. Most of the pitch compensation was used to correct the superfluous $7^{\circ}$ produced by the static offset wedge. (Scan head yaw is corrected using an azimuth rotor with $360^{\circ}$ freedom.) Because of this, the instrument scan head was occasionally unable to account for the ISS operational pitch and attain a proper nadir orientation for science events. Through 5 November 2021, the Hexapod was unable to achieve a scan head nadir orientation for 1555 solar occultation opportunities and 255 lunar occultation opportunities with an uncompensated off-nadir error up to about $1.5^{\circ}$. (As another example, the ISS-RapidScat payload experienced similar problems with its forward pitch offset $[26,27])$. The arrival of Nauka in July 2021 means that the ISS is now in the configuration for which the SAGE III static wedge and Hexapod were designed, and thus the attitude offset will be nominal-absent future changes-during the ongoing SAGE III Extended Mission phase.

It should not be assumed that ISS attitude forecasts are highly accurate. Early in the design phase, ISS external remote sensing instruments with strict pointing requirements should consider design features that permit operability over a wider platform pitch or utilize a dynamic pointing platform with a wide range of mobility to appropriately account for possible ISS configuration delays.

\subsection{Hardware: Track Limited Lifetime Mechanisms Early}

Life-cycle design engineering combined with mechanical testing established the reliability of limited lifetime mechanisms over the three-year Prime Mission. As the Prime Mission was unfolding and consideration turned to the Extended Mission, engineering concerns arose about the operational failure to track the number of cycles executed by limited lifetime mechanisms such as within the instrument solar attenuator assembly, contamination door assembly, and Hexapod pointing mechanisms over the project life-cycle (Phases D-System Assembly, Integration, Test, and Launch and E-Science Operations [28]).

Finite mechanism cycles need to be allocated towards mission success. Meticulously tracking these cycles enables time-to-failure analyses, operational decision-making and schedule optimizing for producing the best science record over the duration of the mission, and correlations of mechanism health and status telemetry with mechanism use. A review of SAGE III Phase D procedures and data was necessary to establish total cycles. On an operational level, limited lifetime mechanisms should be identified early, expected total lifetime cycles should be clearly documented, and a record of the number of executed cycles on the mechanisms should be established beginning in Phase D making use of traveler $\log$ books.

\subsection{Hardware: Visiting Vehicles Introduce the Most Molecular Contamination}

The SAGE III grating spectrometer measures light within the spectral range $280 \mathrm{~nm}$ to $1037 \mathrm{~nm}$ at approximately $1 \mathrm{~nm}$ resolution, but retrievals in the ultraviolet (UV) are particularly sensitive to contamination of the optical train. Therefore, a contamination door that contains a quartz optical window can be closed over the telescope aperture during periods of enhanced external contaminant flux. This optically transparent window permits continued science event acquisition during periods of enhanced contamination at an acceptably diminished signal-to-noise ratio, which is expected to decline with ongoing 
accretion of contaminant material. To date, this impact has been short-term, and science quality through the window returns to baseline performance after a contamination source is removed and both spontaneous desorption and atomic oxygen scrubbing from the orbital ram direction remove material from the low-affinity quartz surface. The contamination door continues to prevent deposition of external contaminants into the instrument telescope. When contamination returns to a tolerable flux, the contamination door is opened to retrieve the best science measurements.

Two Contamination Monitoring Packages (CMPs) consisting of eight Mark 24 Thermoelectric Quartz Crystal Microbalances (TQCMs) from QCM Research provide characterization and redundant monitoring of contaminant deposition from the $2 \pi$ steradian solid angle on the payload side of the ExPRESS Payload Adapter. CMP data are closely examined by the SAGE III mission operations team to determine when the contamination door should remain closed during science events and in what relatively clean direction the instrument assembly azimuth scan head should stow when not acquiring science measurements. Additionally, should the CMPs indicate an unacceptable contaminant flux, the flight computer will close the contamination door as part of the automatic fault detection system. Along with spectrometer measurements of the quartz window's transmission, the payload CMPs enable auditing of the mission contamination budget and correlations with performance changes in the payload thermal control surfaces. The process of Thermogravimetric Analysis (TGA) can be used to help identify chemical constituents accreted on the CMP sensors and to burn off contamination.

To identify sources of contamination in the local ISS environment, each SAGE III TQCM sensor faces a unique direction. While molecular contamination in the free molecular flow regime of LEO is dominated by rectilinear motions, it is also subject to redistribution by, for example, energetic solar flux and ISS scattering surfaces. It is essential that fields of view and Clausing conductance factors for all vacuum inlets be well characterized for each TQCM sensor so that accurate fluxes and directions can be retrieved. Tightly controlling the conductance factors for each TQCM sensor should be a system design requirement, and modifications should be considered carefully and designated quality sensitive. Prior to launch it was anticipated that ISS solar panels would be the largest source of contamination. Solar panel contribution to the background contamination over an orbit is coupled to the solar flux term, and exposure to a given TQCM is related to panel articulation. The solar flux variation over an orbit also induces noise on individual CMP sensors by producing a thermal gradient across the sensor. This makes characterizing the contamination from solar panels on their own problematic. However, contamination from the solar panels seems to be no worse than the background contamination flux, and visiting vehicles have been the largest source of attributable contamination measured [24]. To mitigate their effects on the instrument, the contamination door is closed when vehicles arrive and depart. When vehicles not previously observed by the CMPs arrive, the instrument is commanded into a safe mode for contamination characterization to determine future response.

\subsection{Hardware: Measure the Payload Mechanical Environment When Pointing Is Critical}

Unlike its predecessors, the SAGE III/ISS instrument payload encounters off-nadir attitudes along with jitter conditions caused by the mechanical instability of the ISS environment. The retrieval algorithm for the SAGE III occultation science data product requires precise and accurate knowledge of the instrument boresight pointing with respect to Earth and the radiant target, either Sun or Moon. Shock or random vibration loading on the ISS structure energizes internal modes that translate directly into rapid attitude deviations of the payload from the reported ISS body-fixed coordinate system. Without knowledge of the attitude drifts at the instrument scan head and the associated mechanical jitter, atmospheric data retrieval quality is reduced. Limb scatter science events that measure the scattering of solar radiation by Earth's lit atmosphere do not track a celestial target and therefore do not use the same method to register altitude, latitude, and longitude of retrievals as solar and 
lunar occultation events. To accurately account for pointing during limb events an absolute attitude product of the ExPRESS Logistics Carrier-4 (ELC-4) is needed.

A subsystem on the payload designated as the Disturbance Monitoring Package (DMP), comprising a Miniature Inertial Measurement Unit (MIMU) built by Honeywell Aerospace of Clearwater, Florida, was added to quantify mechanical disturbances. Employing three highly sensitive Honeywell GG1320 Ring Laser Gyroscopes (RLGs), the DMP measures angular attitude rates in three orthogonal axes and reports integrated angular position at $200 \mathrm{~Hz}$. By calibrating to the ISS body coordinate frame on orbit and using attitude determination algorithms, the pointing information needed for post-processing solar, lunar, and limb science is derived, ensuring that the data retrievals maintain the high standard expected of this class of instrument.

The ISS mechanical transfer function between the DMP and the instrument scan head was not assessed during AI\&T. Planned vibration testing originally included a LASER vibrometer characterization of the (linearized) transfer function but was de-scoped because of schedule prioritization. To extend utilization of the DMP to correct (or filter) science data and boresight pointing for the ISS mechanical environment, the phase and amplitude response of the measured DMP vibration that is transmitted to the scan head and scan mirror needs to be understood. The mechanical transfer function can only practically be measured on the ground, and the DMP team has required considerable time to derive a suitable transfer function from science data observations. De-scope will occur, but it is important to recognize that de-scoped characterization testing has mission operational consequences only distantly related to the hardware delivery goals of AI\&T.

\subsection{Software: Flight Projects Need Dedicated IT System Administrators}

Even small flight projects accumulate copious quantities of servers and client systems over time, requiring patches, new software, hard-drive replacements, etc. As the network of computers grows, upkeep falls to existing personnel. In the case of SAGE III, this became a burden on the Ground Systems team. Projects only run as smoothly as their supporting hardware and software. If these activities grow to a full-time job, existing personnel will likely shoulder this responsibility on top of nominal work priorities. A dedicated team of Information Technology (IT) system administrators is advantageous because they understand the latest security concerns and system vulnerabilities and are able to dedicate their time to keeping the systems updated and running.

\subsection{Mission Operations: The International Space Station Offers a Dynamic Environment}

New circumstances serially arise on the ISS. Brand new vehicles arrive bringing novel contaminant fluxes and attitude equilibria, off-nominal crew or robotic operations require flexible mission planning or unexpectedly block science measurements and initiate inquiries by the hosted payload team into root cause, and other emergent circumstances can interrupt daily operations. For example, failure of an ISS BCDU in the ELC-4 power system shut off operational power to the SAGE III instrument payload for one month. Survival power remained available during that time.

These operational challenges imply the need for a relatively sizeable team of mission operations engineers. External ISS payloads should expect that more coverage is needed for mission operations tasks than for free-flying instruments.

\subsection{Mission Operations: Establish a System Early for Trending All Telemetry}

Long-term trending of on-orbit data and telemetry has been a considerable effort. Development and several iterations of the trending product occurred late-beginning in Phase E and requiring significant Phase D reachback to subject matter experts. It is essential to establish a data and telemetry trending protocol along with actionable metrics and a periodic review timeline to monitor the health and reliability of the instrument payload.

Preparation is key. Subject matter experts are the best personnel to determine what data should be trended and how the data are displayed. Trending algorithms, visualiza- 
tions, and metrics should be established early in Phase D. Deliverables should be built into the AI\&T timeline for this development. A centrally-managed document should direct the trending effort and enumerate metrics.

\subsection{Personnel: Hands-On Training Is Essential for New Operators}

Initial mission operator training requirements for the Flight Operations Review were extensive and included many hands-on hours with the flight payload prior to launch. As new team members were added to accommodate personnel turnover, general training packages introduced them to the subsystems. However, this does not replace hands-on experience with flight hardware.

The SAGE III Spread System laboratory allows new operators to run procedures in a high-fidelity, simulated environment without any risk of mistreating the active mission payload. Mission operations is highly process-driven and refined. A flight project should be prepared to supply new personnel with an appropriate level of training to properly prepare them for action. Extensive hours running nominal and off-nominal procedures in a realistic development environment are necessary for operators to properly mature their capabilities.

\section{Stratospheric Aerosol and Gas Experiment IV (SAGE IV) Instrument}

The Stratospheric Aerosol and Gas Experiment IV (SAGE IV) concept is a solar occultation imager CubeSat capable of measuring ozone, aerosols, and other trace gas species at the same quality as previous SAGE instruments (including SAGE III/ISS). The SAGE IV instrument adapts successful ideas from its predecessors but with a modified approach to fit inside of a 6U CubeSat bus as is seen in Figure 1. The methodology has been transformed from a legacy solar-scanning technique with a grating spectrometer to imaging of the entire solar disk directly. Direct imaging is now possible through increased accuracy in CubeSat pointing and higher data rates from LEO. SAGE IV is capable of providing continuity for critical data products, particularly ozone and aerosol in the stratosphere, as described in the 2017 Decadal Survey for Earth Science and Applications from Space [13], albeit at a significantly reduced size and cost. Table 1 contains the species-dependent precision and vertical resolution requirements for the mission.

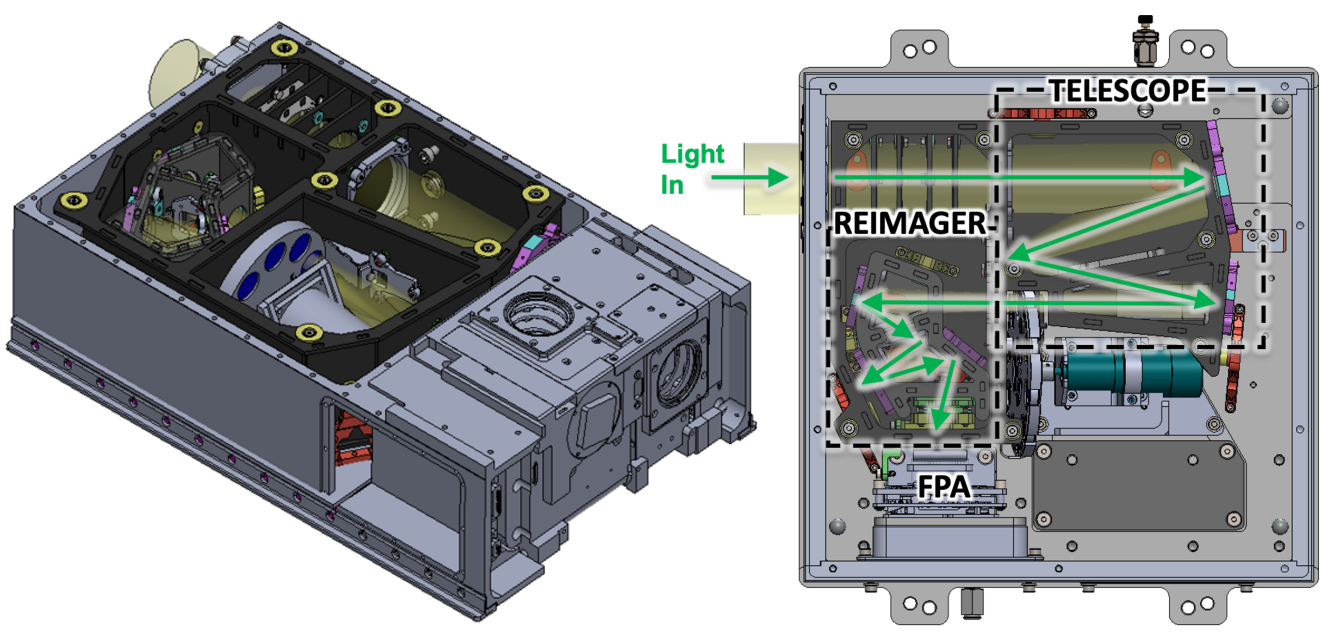

Figure 1. Illustration of the Stratospheric Aerosol and Gas Experiment IV (SAGE IV) instrument concept in a $6 \mathrm{U}$ CubeSat on the left and the optical train on the right. 
Table 1. Science requirements.

\begin{tabular}{cccc}
\hline Species & Altitude Range & Best Precision & Vertical Resolution \\
\hline Ozone & $0 \mathrm{~km}^{*}-70 \mathrm{~km}$ & $1 \%$ & $1 \mathrm{~km}$ \\
\hline Aerosol Extinction & $0 \mathrm{~km}^{*}-40 \mathrm{~km}$ & $5 \%$ & $1 \mathrm{~km}$ \\
\hline Water Vapor & $0 \mathrm{~km}^{*}-60 \mathrm{~km}$ & $10 \%$ & $2 \mathrm{~km}$ \\
\hline Nitrogen Dioxide & $10 \mathrm{~km}^{*}-50 \mathrm{~km}$ & $5 \%$ & $1 \mathrm{~km}$ \\
\hline *Stated altitude or cloud-top, whichever is higher. & &
\end{tabular}

The SAGE IV Pathfinder instrument was funded by the NASA Earth Science Technology Office (ESTO) in 2017 under an Instrument Incubator Program (IIP) to develop a ground demonstration prototype. Taking lessons learned from a long heritage of SAGE instruments, a SAGE IV solar occultation imager concept was developed that could still meet critical atmospheric data continuity needs in the coming decades, but at an order-of-magnitude reduction in cost relative to prior missions. Significant cost savings in instrument and mission architecture enable sustainability of the measurements through repeated deployment of less expensive instruments over time. The smaller form-factor and reduced cost also allow for a small constellation of CubeSats for increased geospatial sampling and increased synergy with other instruments using different observing techniques. The IIP goal was to deliver an instrument prototype that met the science requirements of a potential future flight instrument while only selecting components that have a flight analogue or a clear path to space. Currently, no SAGE IV instrument is funded for a flight mission.

\subsection{Optical Layout}

The apparent solar disk from Earth is $\approx 32$ arcminutes in diameter, and the science field of view $(\mathrm{FOV})$ encompasses $\approx 40$ arcminutes. To add a margin for pointing uncertainty for a future on-orbit mission, the total FOV was required to be $\approx 60$ arcminutes $\left(1^{\circ}\right)$ across. To satisfy vertical resolution requirements, the instantaneous FOV of an individual detector pixel had to be no more than 0.5 arcminutes, which corresponds to 30 microns at the focal plane (i.e., the pitch of a single pixel) for the detector employed in the delivered IIP instrument. In addition, to utilize spectral filters for distinct science channels, the telescope had to afford a location where collimated light could be passed through those filters. Beyond these requirements, care was taken during the design process of the telescope to ensure the best possible performance. Solar occultation measurements routinely span 4-5 orders of magnitude in brightness, and switching from scanning to imaging means that the full range would be contained in a single image. The dynamic range in the observations means that mitigating stray light (i.e., image ghosting, out-of-field stray light, and in-field stray light) is of paramount importance. Furthermore, the telescope needed to maintain these performance requirements throughout the on-orbit thermal environment that could be encountered. Lastly, like previous SAGE instruments, the optical train would require an attenuator to block the majority $(\approx 99 \%)$ of light from the Sun to prevent saturating the detector, but the spectrally dependent solar attenuator's final throughput is determined as the last step to optimize the overall throughput of the system.

Key optical requirements that drove the design of the multispectral imager include (1) image quality, measured as encircled energy at the focal plane, (2) out-of-field stray light rejection, and (3) suppression of ghost images from Fresnel reflections. As image quality and encircled energy are a function of in-field stray light, only unobscured systems were considered with the largest reasonable aperture that could fit within a CubeSat as shown in Figure 1. It is expected that there will be meaningful temporal and spatial thermal gradients during operations caused by periodic solar flux and the small size of the host spacecraft and as such, the selection of thermally insensitive materials for each of the primary component types (metering structure, mounts, and optics) is critical to maintaining alignment through the range of operating conditions, as well as ensuring the structural survivability during launch. Exhaustive materials trade studies were performed to understand which metering 
structure material would perform best (i.e., find a cost-effective solution that satisfies requirements), and ultimately a carbon fiber metering structure, with Invar-36 mounts and ZERODUR ${ }^{\circledR}$ mirrors was selected [29].

The amount of stray light incident on the Focal Plane Array (FPA) because of in-field or out-of-field scattering and ghost reflections was quantified to verify that it would not be strong enough to corrupt the science image. The stray light requirement is defined by no greater than a relative signal strength of $10^{-4}$, which was exceeded by a performance of a maximum contribution of $10^{-5}$, and the ghost reflection requirement is defined by no greater than a relative contribution of $10^{-3}$ and to be outside the FOV by at least $1^{\circ}$, which was also met with a performance of $5^{-4}$. Testing performed at the Space Dynamics Laboratory (SDL) of Utah State University (USU) stray light testing facility provided a strong correlation between the measurements and conservative simulations and modeling. The single and unavoidable ghost introduced by the wedged attenuator showed up out of field where expected and agreed with modeling, with a marginal variation in magnitude [29]. Because of the success of the measurements and their agreement with simulation, the model has been validated ensuring a successful flight design provided good qualification of components is achieved. Encircled energy within the essentially diffraction limited telescope was measured to be between $92.4 \%$ and $94.2 \%$ within an individual pixel area across the image plane, which exceeded requirements and was within the predicted as-built performance range, including manufacturing tolerances.

\subsection{Filter Wheel Assembly}

The imaging technique necessitated the use of spectral interference filters, since it is impossible to simultaneously image and perform spectroscopy without a complicated design. A similar approach — of using filters for different science channels—was used by the highly successful SAGE II instrument during its 21 years of operation. For SAGE IV, the decision was made to incorporate a filter wheel into the design. The wheel itself would need to fit within a CubeSat (i.e., $<10 \mathrm{~cm}+$ margin), while incorporating as many filter locations as possible to maximize the number of observable species. To achieve science and onboard characterization goals (e.g., dark current and flat-fielding), the filter wheel would need at least 9 locations. With a sampling frequency requirement of one image at each wavelength of interest every two seconds, the wheel would need to spin at $0.5 \mathrm{~Hz}$. The size of each filter is a coupled requirement with the telescope design (beam width at filtering location) and detector timing to allow for adequate integration time through the clear aperture of each filter as the filter wheel rotates without obscuring the light or introducing vignetting. Lastly, to ensure detector integration is appropriately timed, the rotational location of the filter wheel would need to be known to within the timing limits of the ability to readout the detector. Regarding this design choice, it is worth noting that, while the addition of a moving part is not ideal, alternatives (such as an acoustic optical tunable filter) that met our form, fit, and functional requirements were not available at the beginning of the development process. Naturally, this design choice may be revisited in the future as available technology advances.

The SAGE IV Filter Wheel Assembly (FWA) is designed to house eight transmissive bandpass filters and filter/diffuser combinations along with one opaque flat mirror. The filter wheel and motor are supported by the motor mount, which holds the full assembly at a $7.5^{\circ}$ angle from the chief ray to direct Fresnel reflections into a beam dump. The assembled filter wheel is shown in Figure 2. Each of the nine positions in the filter wheel is either a science channel specifically chosen to observe a target species or as a characterization channel chosen to enable characterization of the instrument's performance on-orbit. To enable measurements of detector dark current and telescope background during science observations, one spot within the wheel has a counter-bore with a flat mirror inserted. The counter-bore ensures that no light passes through and that the mirror is representative of the interference filters that reflect the majority of incident light-the out-of-band portionback to the telescope's beam dump. With a "light block" as an element in the filter wheel, 
both a dark current image and a background image of critical surface stray light can be taken as part of the standard $0.5 \mathrm{~Hz}$ data collection, and potential thermal transient effects can be observed, characterized, and removed. The spectral locations of the bandpass filters used for the delivered IIP system are similar to those used for the SAGE II mission and are detailed in Table 2.

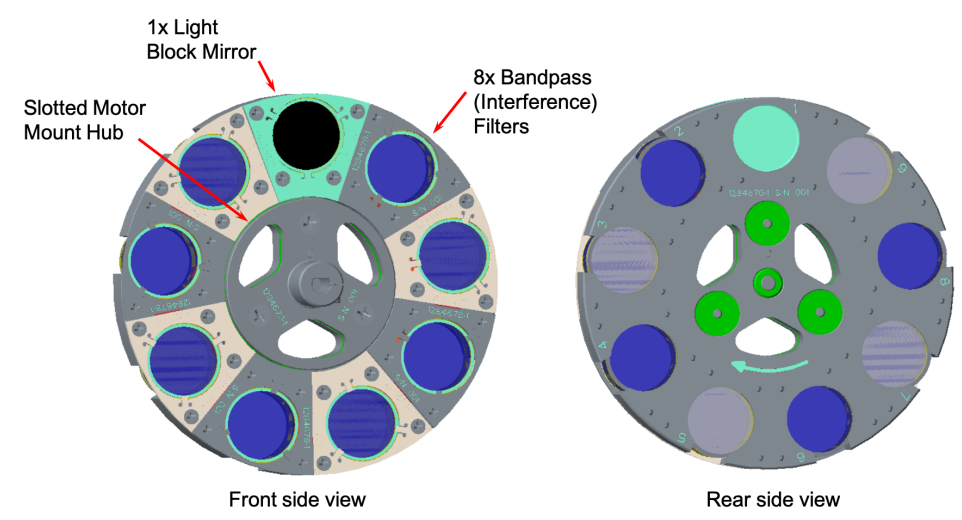

Figure 2. SAGE IV filter wheel with filters, diffuser, and light block.

Table 2. Bandpass filters in the FWA.

\begin{tabular}{ccc}
\hline Center Wavelength & Bandwidth & Target Species \\
\hline $386 \mathrm{~nm}$ & $15 \mathrm{~nm}$ & Aerosols/Blue-end Response \\
$448 \mathrm{~nm}$ & $2 \mathrm{~nm}$ & Nitrogen Dioxide \\
$452 \mathrm{~nm}$ & $2 \mathrm{~nm}$ & Nitrogen Dioxide \\
$525 \mathrm{~nm}$ & $15 \mathrm{~nm}$ & Aerosols \\
$600 \mathrm{~nm}$ & $15 \mathrm{~nm}$ & Ozone \\
$1020 \mathrm{~nm}$ & $15 \mathrm{~nm}$ & Aerosols/Red-end Response \\
\hline
\end{tabular}

In addition to the "light block", diffusers are the other characterization element within the filter wheel. Where two (or more) pixels on a detector look at different spatial scenes and need to be compared, a pixel-to-pixel cross-calibration or "flat-fielding" correction will need to be applied to the data to account for spatial non-uniformities in pixel responsivity. This is a standard step in scientific image processing that is often not required for simple pushbroom spectrometers. While flat-fielding is easily characterized in the laboratory, this behavior will change on orbit from radiation displacement damage and might also be susceptible to minor changes in temperature. As such, it is necessary to perform this kind of instrument characterization operationally in outer space. For a highly sensitive measurement such as solar occultation, observing the solar disk through a diffuser can provide a sufficiently uniform source to perform pixel-to-pixel cross-calibrations. Incorporating such a characterization measurement into the filter wheel has the added bonus of observing how pixel response differs with changes in the thermal profile of the sensorcraft through extended exoatmospheric measurements. Such thermal transients have been shown to be potentially problematic and difficult to characterize for previous SAGE incarnations.

\subsection{Detector}

The SAGE IV detector is key to meeting the measurement requirements shown in Table 1. Water vapor is the most difficult species to measure and requires a Signal-to-Noise Ratio (SNR) of $\approx 2000$ over a $30 \mu \mathrm{m} \times 30 \mu \mathrm{m}$ spot (i.e., the size of an individual pixel). The large SNR requirement is in a shot-noise-limited regime (i.e., where the noise follows Poisson statistics and is equivalent to the square root of the signal) and so to achieve a SNR of $\approx 2000$, a pixel on the detector would need a minimum well-depth of 4 million electrons in the linear range. It was assumed the detector would be linear when the well-depth was between $10 \%$ and $90 \%$ full; therefore, the detector's well depth would need to be $25 \%$ 
larger than the 4 million electrons for a total of 5 million electrons. The desired spectral range in the UV/VIS/NIR, the deep well-depth required, and a desire to have low darkcurrent at ambient temperatures (no significant cooling required) compelled the use of silicon as the photosensitive substrate. Silicon requires a stable temperature during the few minutes encompassed by an occultation measurement, which is possible through either a Thermoelectric Cooler (TEC) or a heater, and does not require cryocooling. In addition, for the imaging concept to work, each pixel must be read out individually, requiring a PIN diode array such as in a Complementary Metal Oxide Semiconductor (CMOS) sensor, as opposed to a typical Charge-Coupled Device (CCD) read out. The telescope produces an image of the full FOV of roughly $3.6 \mathrm{~mm} \times 3.6 \mathrm{~mm}$ at the focal plane that must be captured by an array of pixels. The number and pitch of pixels are unimportant provided the effective SNR is achievable over the $30 \mu \mathrm{m} \times 30 \mu \mathrm{m}$ spot through the binning of pixels.

\subsection{Sunlook Testing}

To evaluate the operational performance of the SAGE IV IIP instrument an equatorial mount carrying a surrogate $6 \mathrm{U}$ CubeSat chassis were deployed outdoors to house the Pathfinder instrument for extended Sunlook testing over several months. These tests rigorously examined telescope, filter wheel, and detector performances while tracking the Sun terrestrially during daylight hours. The surrogate chassis was placed under dry nitrogen purge to test thermoelectric control of the focal plane temperature. Embedded software and firmware along with telemetry, command, and control through the ground systems were proven, including fine tracking of the solar target via closed-loop instrument commanding of the equatorial mount. The image data met science requirements for image quality, signal, and noise characteristics and the system was shown to function as desired after a few technical challenges were resolved [30]. For example, driving of the focal plane TEC with Pulse-Width Modulation (PWM) directly from embedded firmware, while more power efficient than using a DC driving current, proved to radiate too much noise from the Peltier element directly at the detector. Consequently, an analog DC driver has replaced the embedded firmware PWM driver. Sunlook testing greatly increases confidence in flight mission success, and laboratory testing and characterizations have continued subsequent to Sunlook.

\section{Summary}

The ISS is a powerful and unique platform that can offer many advantages to scientific payloads. Every spaceborne platform comes with challenges; however, future payloads can utilize the lessons learned from prior experiments, such as those presented from SAGE III/ISS, to leverage the full benefits of the space station. A series of technological advances have made the transition from SAGE III/ISS to SAGE IV possible, and the corresponding cost savings should enable the sustainability of the measurements into the future. A complete SAGE IV Pathfinder prototype instrument system that meets SAGE science requirements and has a clear path to flight was delivered to NASA and rigorously tested through IIP funding from ESTO. While SAGE IV is not yet funded for a flight mission, the authors are hopeful that it will be in the near future so that SAGE can continue its legacy of aerosol, ozone, and water vapor measurements.

Author Contributions: C.A.H. is the Deputy Project Scientist for SAGE III/ISS and provided insight on lessons learned. R.D., C.A.H. and J.P.L. were all integrally involved in the SAGE IV IIP and equally contributed to the development of the instrument and the writing of those sections. All authors have read and agreed to the published version of the manuscript.

Funding: SAGE III/ISS is a NASA Langley managed mission funded by the NASA Science Mission Directorate within the Earth Systematic Mission Program. Enabling partners are the NASA Human Exploration and Operations Mission Directorate, International Space Station Program and the European Space Agency. The SAGE IV concept has been developed through the NASA Earth Science Technology Office Instrument Incubator Program (IIP-16-0077) with additional support from NASA Langley Research Center. 
Institutional Review Board Statement: Not applicable.

Informed Consent Statement: Not applicable.

Data Availability Statement: Not applicable.

Conflicts of Interest: The authors declare no conflict of interest.

\section{References}

1. Chu, W.; McCormick, M. Inversion of stratospheric aerosol and gaseous constituents from spacecraft solar extinction data in the 0.38-1.0- $\mu \mathrm{m}$ wavelength region. Appl. Opt. 1979, 18, 1404-1413. [CrossRef] [PubMed]

2. McCormick, M.P.; Hamill, P.; Pepin, T.J.; Chu, W.P.; Swissler, T.J.; McMaster, L.R. Satellite Studies of the Stratospheric Aerosol. Bull. Am. Meteorol. Soc. 1979, 60, 1038-1047. [CrossRef]

3. WMO. Atmospheric Ozone 1985; Global Ozone Research and Monitoring Project-Report No. 16; World Meteorological Organization: Geneva, Switzerland, 1985.

4. Mauldin III, L.; Zaun, N.; McCormick, M., Jr.; Guy, J.; Vaughn, W. Stratospheric Aerosol and Gas Experiment II instrument: A functional description. Opt. Eng. 1985, 24, 307-312. [CrossRef]

5. Damadeo, R.P.; Zawodny, J.M.; Thomason, L.W.; Iyer, N. SAGE version 7.0 algorithm: Application to SAGE II. Atmos. Meas. Tech. 2013, 6, 3539-3561. [CrossRef]

6. WMO. Scientific Assessment of Ozone Depletion: 2018; Global Ozone Research and Monitoring Project-Report No. 58; World Meteorological Organization: Geneva, Switzerland, 2018.

7. McCormick, M.P.; Thomason, L.W.; Trepte, C. Atmospheric Effects of the Mt. Pinatubo Eruption. Nature 1995, 373, 399-404. [CrossRef]

8. Chu, W.P.; McCormick, M.P.; Zawodny, J.M.; Mauldin, L.E., III. Stratospheric Aerosol and Gas Experiment III. In Proceedings of the Earth Observing Systems II. International Society for Optics and Photonics, San Diego, CA, USA, 18 September 1997; Volume 3117, pp. 11-18. [CrossRef]

9. Wang, H.J.R.; Damadeo, R.; Flittner, D.; Kramarova, N.; Taha, G.; Davis, S.; Thompson, A.M.; Strahan, S.; Wang, Y.; Froidevaux, L.; et al. Validation of SAGE III/ISS Solar Occultation Ozone Products With Correlative Satellite and Ground-Based Measurements. J. Geophys. Res. Atmos. 2020, 125, e2020JD032430. [CrossRef]

10. Chen, Z.; Bhartia, P.K.; Torres, O.; Jaross, G.; Loughman, R.; DeLand, M.; Colarco, P.; Damadeo, R.; Taha, G. Evaluation of OMPS/LP Stratospheric Aerosol Extinction Product Using SAGE III/ISS Observations. Atmos. Meas. Tech. 2020, 13, 3471-3485. [CrossRef]

11. Davis, S.M.; Damadeo, R.; Flittner, D.; Rosenlof, K.H.; Park, M.; Randel, W.J.; Hall, E.G.; Huber, D.; Hurst, D.F.; Jordan, A.F.; et al. Validation of SAGE III/ISS Solar Water Vapor Data With Correlative Satellite and Balloon-Borne Measurements. J. Geophys. Res. Atmos. 2021, 126, e2020JD033803. [CrossRef]

12. Park, M.; Randel, W.J.; Damadeo, R.P.; Flittner, D.E.; Davis, S.M.; Rosenlof, K.H.; Livesey, N.; Lambert, A.; Read, W. Near-Global Variability of Stratospheric Water Vapor Observed by SAGE III/ISS. J. Geophys. Res. Atmos. 2021, 126, e2020JD034274. [CrossRef]

13. National Academies of Sciences, Engineering, and Medicine. Thriving on Our Changing Planet: A Decadal Strategy for Earth Observation from Space; The National Academies Press: Washington, DC, USA, 2018. [CrossRef]

14. Clean Air Act of 196342 U.S.C. § 7401 et seq. (As Amended 1990, Title VI, 42 U.S.C. § 7671b). Available online: https: / / www.epa.gov / clean-air-act-overview / evolution-clean-air-act (accessed on 26 September 2021).

15. Solomon, S.; Portmann, R.W.; Garcia, R.R.; Thomason, L.W.; Poole, L.R.; McCormick, M.P. The role of aerosol variations in anthropogenic ozone depletion at northern midlatitudes. J. Geophys. Res. Atmos. 1996, 101, 6713-6727. [CrossRef]

16. Solomon, S.; Daniel, J.S.; Neely, R.R.; Vernier, J.P.; Dutton, E.G.; Thomason, L.W. The Persistently Variable "Background" Stratospheric Aerosol Layer and Global Climate Change. Science 2011, 333, 866-870. [CrossRef] [PubMed]

17. Schmidt, A.; Robock, A. Volcanism, the atmosphere and climate through time. In Volcanism and Global Environmental Change; Schmidt, A., Fristad, K., Elkins-Tanton, L., Eds.; Cambridge University Press: Cambridge, UK, 2015; pp. 195-207. [CrossRef]

18. IPCC. 2014: Climate Change 2014: Synthesis Report. In Contribution of Working Groups I, II and III to the Fifth Assessment Report of the Intergovernmental Panel on Climate Change; Core Writing Team, Pachauri, R.K., Meyer, L.A., Eds.; IPCC: Geneva, Switzerland, 2014; 151p.

19. Jacob, D.J. Introduction to Atmospheric Chemistry; Princeton University Press: Princeton, NJ, USA, 1999; ISBN 978-1400841547.

20. Mote, P.W.; Rosenlof, K.H.; McIntyre, M.E.; Carr, E.S.; Gille, J.C.; Holton, J.R.; Kinnersley, J.S.; Pumphrey, H.C.; Russell, J.M., III; Waters, J.W. An atmospheric tape recorder: The imprint of tropical tropopause temperatures on stratospheric water vapor. $J$. Geophys. Res. Atmos. 1996, 101, 3989-4006. [CrossRef]

21. Rosenlof, K.H.; Tuck, A.F.; Kelly, K.K.; Russell, J.M., III; McCormick, M.P. Hemispheric asymmetries in water vapor and inferences about transport in the lower stratosphere. J. Geophys. Res. Atmos. 1997, 102, 13213-13234. [CrossRef]

22. Randel, W.J.; Wu, F.; Russell, J.M., III; Roche, A.; Waters, J.W. Seasonal Cycles and QBO Variations in Stratospheric CH4 and H2O Observed in UARS HALOE Data. J. Atmos. Sci. 1998, 55, 163-185. [CrossRef]

23. Randel, W.; Park, M. Diagnosing Observed Stratospheric Water Vapor Relationships to the Cold Point Tropical Tropopause. J. Geophys. Res. Atmos. 2019, 124, 7018-7033. [CrossRef] 
24. Dawson, T.T.; Hill, C.A.; Rowell, A.F.; Leavor, K.R.; Hawley, S.A. SAGE III/ISS Contamination Monitoring Package: Observations in Orbit; Technical Report NASA/TM-20205001963; National Aeronautics and Space Administration, Langley Research Center: Hampton, Virginia, 2020. Available online: https:/ / ntrs.nasa.gov/ citations/20205001963 (accessed on 26 September 2021).

25. SAGE III on ISS Version 5.1 Release Notes. 2020. Available online: https://asdc.larc.nasa.gov/documents/sageiii-iss/guide/V5 .1ReleaseNotes.pdf (accessed on 26 September 2021).

26. Havens, G.; Boland, S.; Stiles, B.; Poulsen, L.; Perkovic-Martin, D. RapidScat First Year of Operations. In Proceedings of the ISS Payload Operations and Integration Working Group (POIWG \#38), Huntsville, Alabama, 20 July 2015.

27. Madsen, N.M.; Long, D.G. Calibration and Validation of the RapidScat Scatterometer Using Tropical Rainforests. IEEE Trans. Geosci. Remote. Sens. 2016, 54, 2846-2854. doi: 10.1109/TGRS.2015.2506463. [CrossRef]

28. NASA. NASA Space Flight Program and Project Management Handbook; NPR 7120.5E; National Aeronautics and Space Administration: Washington, DC, USA, 2014.

29. Halterman, A.C.; Damadeo, R.; Hill, C.; Buleri, C.; Murchison, L.; Obland, M.; Phenis, A.; Yacoby, S. SAGE IV Pathfinder multi-spectral imaging spectrometer telescope paves the way for semi-custom CubeSat imaging missions. In Proceedings of the Algorithms, Technologies, and Applications for Multispectral and Hyperspectral Imagery XXV, Baltimore, MD, USA, 16-18 April 2019; International Society for Optics and Photonics: Baltimore, MD, USA, 2019; Volume 10986, pp. 125-139. [CrossRef]

30. SAGE IV Pathfinder IIP Final Report in Preparation and Will Be on 2022. Available online: https://ntrs.nasa.gov/oncecomplete (accessed on 26 September 2021). 